\title{
Role of Pressure in Organic Fouling in Forward Osmosis and Reverse Osmosis
}

\author{
Journal of Membrane Science
}

Revised: July 13, 2015

\footnotetext{
Ming Xie ${ }^{1,2}$, Jongho Lee ${ }^{1}$, Long D. Nghiem ${ }^{2}$, and Menachem Elimelech ${ }^{1 *}$

${ }^{1}$ Department of Chemical and Environmental Engineering, Yale University, New Haven, CT 06520-8286, United States

${ }^{2}$ Strategic Water Infrastructure Laboratory, School of Civil, Mining and Environmental Engineering, University of Wollongong, Wollongong, NSW 2522, Australia
}

*Corresponding author. E-mail: menachem.elimelech@yale.edu 


\begin{abstract}
Fundamental understanding of membrane fouling in osmosis-driven membrane processes is important for further deployment of this emerging technology in desalination and wastewater reuse. In this study, we investigated the role of pressure in organic fouling and reversibility in forward osmosis (FO) and reverse osmosis (RO) using alginate as a model organic foulant. Varying contributions of pressure (i.e., osmotic versus hydraulic) to the overall driving force were realized in forward osmosis (FO), pressure-assisted FO (PFO), and reverse osmosis (RO) experiments, while the same total driving force for water permeation was applied. Confocal laser scanning microscopy (CLSM) was used to examine alginate fouling layer structure in the hydrated state, which informed two key parameters: fouling layer thickness and foulant volume. We observed that the resulting fouling layer became increasingly more compact in the order of FO, PFO, and RO experiments. Fouling layer reversibility followed the same trend, with the highest and lowest reversibility observed for the FO and RO fouling experiments, respectively. Possible mechanisms for fouling layer compaction in RO were discussed, including permeate drag force and foulant compressibility, as opposed to FO where only permeate drag force applies. Our findings suggest that pressure mechanistically alters the membrane fouling layer structure and fouling reversibility, leading to higher fouling reversibility in FO, where the driving force is an osmotic pressure, than RO, where the driving force is hydraulic pressure.
\end{abstract}

Keywords: Forward osmosis; Reverse osmosis; Fouling reversibility; Fouling layer compaction; Fouling layer structure 


\section{Introduction}

Reverse osmosis (RO), a pressure-driven membrane process, is a reliable technology to augment water supply through seawater desalination and wastewater reuse [1]. The RO process uses hydraulic pressure as the driving force for water permeation through a dense polymeric membrane, which consumes a vast amount of energy for seawater desalination [2]. RO plants require an average of $4 \mathrm{kWh}$ electric energy to produce one cubic meter of freshwater, which is associated with $2.3 \mathrm{~kg} \mathrm{CO}_{2}$ emission from power plants [3]. In both desalination and wastewater reuse applications, RO membrane fouling is inevitable [4, 5], thereby requiring the use of chemical cleaning agents [6] and increasing the cost of water production.

Forward osmosis (FO), an osmosis-driven membrane process, could potentially advance desalination and wastewater reuse. FO utilizes the osmotic pressure of a highly concentrated draw solution as the driving force to transfer water from the feed solution to the draw solution through a dense polymeric membrane. FO has demonstrated a much lower fouling propensity and higher fouling reversibility than $\mathrm{RO}$, which was attributed to the lack of applied hydraulic pressure [7-10]. Consequently, FO is widely used to treat low quality feedwaters, including landfill leachate [11], anaerobic digester concentrate [12], activated sludge solution [13, 14], and municipal wastewater [15-17].

Despite ample experimental reports regarding the lower fouling propensity of FO, it is not clear whether the lack of hydraulic pressure in FO mechanistically alters membrane fouling and cleaning behavior. We previously hypothesized that the fouling layer structure in FO is markedly different from that in $\mathrm{RO}$, with a loose and spare fouling layer in FO and a dense and compact layer in RO [7, 8]. This hypothesis regarding the structure of the fouling layer in FO was

supported by several indirect experimental observations. Lee et al. [10] reported that water flux was almost completely recovered after 'physical' cleaning by increasing the feed cross-flow velocity. In another study, Mi and Elimelech [8] compared water flux recovery in FO and RO after membrane physical cleaning, where water flux recovery in FO was almost $100 \%$, while that in RO was only $70 \%$.

A direct, detailed examination of FO and RO fouling layer structure in the hydrated state holds the key to this fundamental question regarding the role of pressure in FO and RO fouling. Previous attempts were made to observe FO fouling processes directly by optical microscopy, 
involving various foulants, such as algae [18], latex particles [19], and gypsum-alginate mixture [20]. These observations provided information on surface coverage and morphology of FO fouling layers on the membrane surface. However, optical microscopy cannot shed light on the structure, particularly thickness and density, of the fouling layers.

We propose a confocal laser scanning microscopy (CLSM) approach to examine the membrane fouling layer structure in $\mathrm{FO}$ and $\mathrm{RO}$ in the hydrated state at the micrometer scale. CSLM provides information on a fouling layer at different depths of a three-dimensional object as a result of non-invasive optical sectioning of the sample, thereby non-invasively visualizing the fouling layer structure [21, 22]. This approach was successfully applied to quantitatively investigate the structure of organic fouling layers comprising proteins and polysaccharides in microfiltration $[23,24]$.

The aim of this study is to investigate the role of pressure in organic fouling in FO and RO. We employed FO, pressure-assisted FO (PFO), and RO processes to compare alginate fouling behaviors. These membrane processes entail varying contributions of hydraulic pressure to the overall driving force. Water flux decline during fouling and water flux recovery after physical cleaning were measured to elucidate the role of pressure in membrane fouling. Membranes were characterized by CLSM after fouling and physical cleaning in the hydrated state to delineate the fouling layer structure. Possible mechanisms accounting for such different structures under varying contributions of hydraulic pressure are discussed.

\section{Materials and methods}

\subsection{FO membrane and mass transfer parameters}

Commercially available polyamide thin-film composite (TFC) FO membranes (HTI, Albany, OR) were used in this study. The TFC membrane was received as a flat sheet and stored at $4{ }^{\circ} \mathrm{C}$ in deionized (DI) water. Key membrane transport parameters were determined using the method of Tiraferri et al. [25], resulting in water permeability coefficient, $A$, of $1.72 \mathrm{~L} \mathrm{~m}^{-2} \mathrm{~h}^{-1}$ bar $^{-1}$, salt $(\mathrm{NaCl})$ permeability coefficient, $B$, of $0.169 \mathrm{~L} \mathrm{~m}^{-2} \mathrm{~h}^{-1}$, and structural parameter, $S$, of $425 \mu \mathrm{m}$. 


\subsection{FO, RO, and pressure-assisted FO setups}

We employed three laboratory-scale, cross-flow membrane filtration setups: FO, PFO, and RO to elucidate the role of pressure in organic fouling. Details of the experimental setups are provided in Supplementary Data (Figure S1).

All three membrane setups were equipped with membrane cells of identical dimensions (77 $\mathrm{mm} \times 26 \mathrm{~mm} \times 3 \mathrm{~mm}$ ). In all experiments, the temperatures of the solutions were maintained at $20 \pm 0.1^{\circ} \mathrm{C}$ using a chiller/heater (Neslab RTE 7) equipped with stainless steel heat exchanger coils, submerged in stainless steel reservoirs. The average flow rates of the feed solutions in contact with the membrane active layer were monitored by rotameters and kept at $1 \mathrm{~L} / \mathrm{min}$ (corresponding to a cross flow velocity of $8.5 \mathrm{~cm} / \mathrm{s}$ ) to provide identical shear stress on the membrane surface.

In FO experiments, two variable speed gear pumps (Cole-Parmer, Vernon Hills, IL) were used to circulate the feed and draw solutions at the same flow rate $(1 \mathrm{~L} / \mathrm{min})$. The draw solution reservoir was placed on a digital balance (Mettler Toledo Inc., Hightstown, NJ) and the temporal weight changes were recorded by a computer to calculate the permeate water flux.

For the RO setup, the unit was equipped with a positive displacement pump (Wanner Engineering Inc., Minneapolis, MN). Permeate flow was measured by a digital flow meter (Optiflow 1000, Agilent Technologies, Palo Alto, CA) connected to a computer.

For the PFO setup, a variable speed gear pump (Cole-Parmer, Vernon Hills, IL) and a high-pressure positive displacement pump (Hydra-cell, Wanner Engineering, Inc., Minneapolis, $\mathrm{MN}$ ) were used in a closed loop to circulate the feed solution and draw solution, respectively, at the same flow rate $(1 \mathrm{~L} / \mathrm{min})$. Hydraulic pressure was applied to the feed solution while the draw solution was maintained at an ambient pressure. Flow rate and pressure of the feed solution were controlled by adjusting a bypass needle valve and backpressure valve. Water flux through the membrane was measured from the temporal weight change of the draw solution.

\subsection{Experimental procedure of membrane fouling and cleaning}

We compared alginate organic fouling behaviors in FO, RO, and PFO. The same feed solution of $200 \mathrm{mg} / \mathrm{L}$ sodium alginate and $1 \mathrm{mM} \mathrm{CaCl}_{2}$ was used for all fouling experiments [26]. An initial water flux of $16.5 \pm 0.8 \mathrm{~L} \mathrm{~m}^{-2} \mathrm{~h}^{-1}$ was achieved by adjusting the draw solution 
concentration in FO, applied pressure in RO, and both draw solution concentration and applied pressure in PFO experiments. A glucose solution (2.5 M) was used as a draw solution in FO to minimize the impact of reverse draw solute diffusion on membrane fouling [27, 28]. Hydraulic pressure of 12.5 bar (180 psi) was applied in RO, while in PFO, a combined driving force was applied by using $1.5 \mathrm{M}$ glucose draw solution and $5.5 \mathrm{bar}$ ( $80 \mathrm{psi}$ ) of hydraulic pressure in the feed solution. These three sets of fouling experiments provided a unique perspective for observing alginate fouling behaviors under a range of applied hydraulic pressures, but at identical initial water flux. A baseline experiment (i.e., feed without alginate foulants) was carried out for both FO and PFO fouling experiments to correct the flux decline due to the continuous concentration of the feed solution and dilution of the draw solution, as described in Supplementary Data (S2 and Figure S2). Fouling experiments at the conditions described above were carried out for $\sim 23$ hours to obtain $500 \mathrm{~mL}$ cumulative permeate volume.

Membrane physical cleaning was carried out at the conclusion of each fouling experiment. Experimental conditions for membrane physical cleaning in FO, PFO, and RO include a crossflow velocity of $17 \mathrm{~cm} / \mathrm{s}$, DI water, and a cleaning duration of 30 minutes. After the physical membrane cleaning, water flux was measured using alginate foulant-free solution (i.e., 1 $\mathrm{mM} \mathrm{CaCl}_{2}$ ) to determine water flux recovery, under the same aforementioned experimental conditions in membrane fouling.

Water flux recovery, $R$, after physical cleaning is calculated from [29]

$R(\%)=\frac{J_{c}-J_{a}}{J_{b}-J_{a}} 100$

where $J_{\mathrm{b}}$ is the water flux with the background electrolyte solution (i.e., $1 \mathrm{mM} \mathrm{CaCl}_{2}$ ) before fouling, $J_{\mathrm{a}}$ is the water flux with the background electrolyte solution after fouling, and $J_{\mathrm{c}}$ is the water flux with the background electrolyte solution after physical cleaning. The water flux recovery is an indicator for fouling reversibility.

\subsection{Fouling layer characterization}

We employed confocal laser scanning microscopy (CLSM) to characterize the alginate fouling layers in the hydrated state. Membrane subsections were cut $\left(1 \mathrm{~cm}^{2}\right)$ from the center of the alginate-fouled membranes from each experiment. The membrane sections were then placed 
in a petri-dish with $2 \mathrm{~mL}$ feed solution, followed by a staining procedure where $60 \mu \mathrm{L}$ of $50 \mu \mathrm{M}$ concavalin A (Con A, Alexa Flour 633 conjugated, Invitrogen, MA) in $0.1 \mathrm{M} \mathrm{NaHCO}_{3}$ buffer of pH 8.3 were added to identify polysaccharides. After the membrane sections were left in the dark for 30 minutes to allow for polysaccharide-Con A specific binding [30]. The stained membrane samples were rinsed three times with a foulant-free, $1 \mathrm{mM} \mathrm{CaCl}_{2}$ solution to remove any unbound stain, and then mounted in a custom-built characterization chamber for confocal imaging [31].

Confocal images were captured using a CLSM (Zeiss LSM 510, Carl Zeiss, Inc.) equipped with a Plan-Apochromat 20×/0.8 numerical aperture objective. Con A fluorophore was excited with $633 \mathrm{~nm}$ helium-neon laser. A minimum of three $\mathrm{Z}$ stack random fields $(635 \mu \mathrm{m} \times 635 \mu \mathrm{m})$ were collected for each sample, with a slice thickness of $2.3 \mu \mathrm{m}$, using ZEN (Carl Zeiss, Inc.) to obtain a representative ortho-image of fouling layer. The fouling layer dimensions were analyzed by capturing a minimum of ten random $\mathrm{Z}$ stack regions $(90 \mu \mathrm{m} \times 90 \mu \mathrm{m})$ for each sample, with a slice thickness of $1.2 \mu \mathrm{m}$, using ZEN (Carl Zeiss, Inc.).

Image analysis was performed using Auto-PHLIP-ML (http://sourceforge.net/projects/phlip), ImageJ software (http://rsbweb.nih.gov), and MATLAB (The Mathworks, Inc.). Thickness and foulant volume were determined by identifying Con A stained polysaccharides of the fouling layers for all samples, using a Matlab PHLIP code [32]. Thickness represents the spatial size of the alginate fouling layer, while foulant volume is defined as the genuine volume of foulants by multiplying the number of foreground pixels in an image stack by the voxel volume in the hydrated fouling layer per membrane area, thus providing an estimate of the alginate fouling layer density [33]. A more detailed description of the Auto- PHLIP-ML software can be found elsewhere [32, 33].

\section{Results and Discussion}

\subsection{Membrane fouling behavior}

We related membrane fouling behavior, including water flux decline and recovery, to the driving force for water permeation through the membrane (Figure 1). While the FO process only uses osmotic pressure as the driving force, the RO membrane process is driven by pure hydraulic pressure. Uniquely, the PFO membrane filtration combines osmotic and hydraulic pressures as the driving force. 

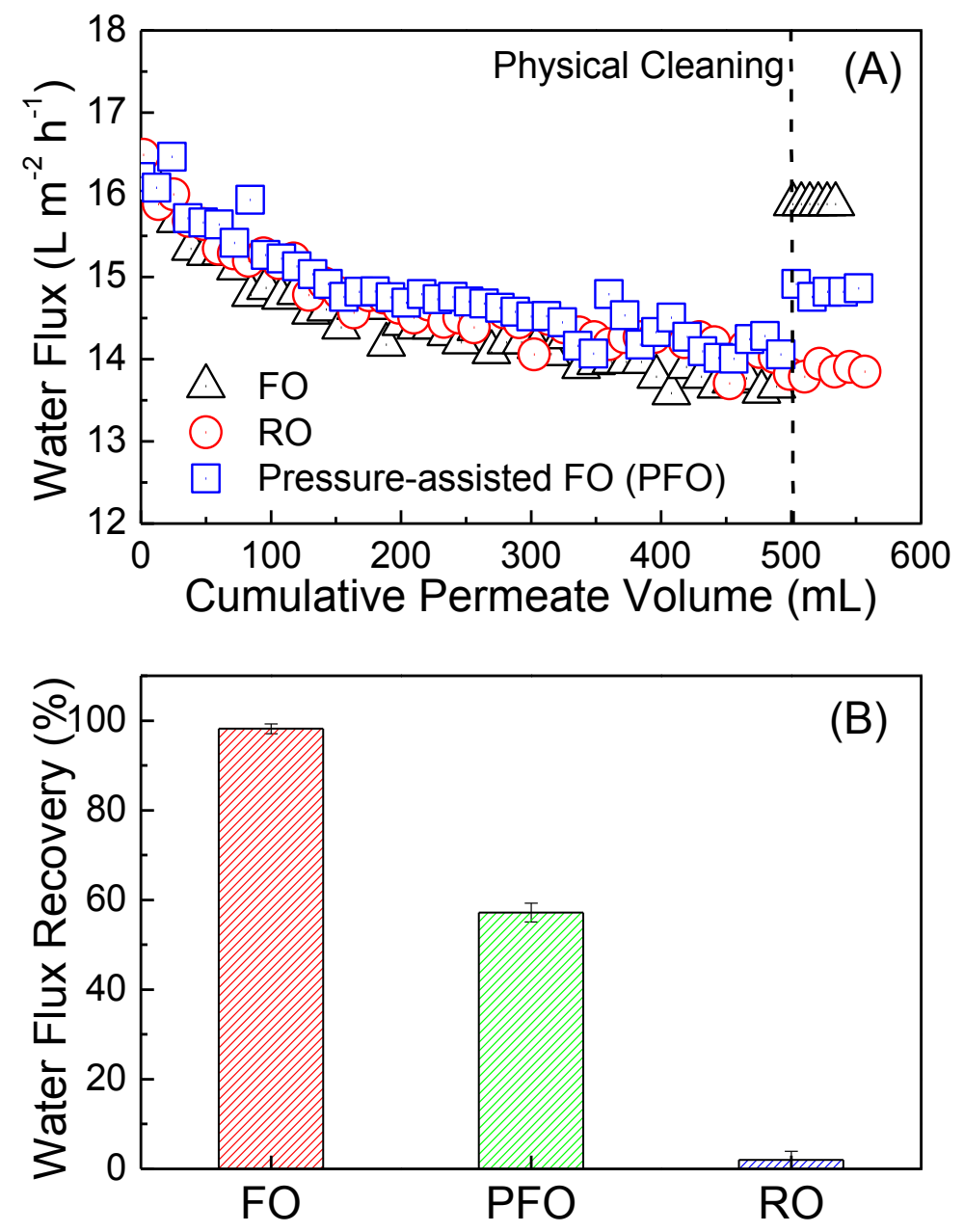

Figure 1: (A) Water flux decline during organic fouling and (B) water flux recovery after physical cleaning of fouled membrane in FO, pressure-assisted FO (PFO), and RO. Fouling experimental conditions were: feed solution contained $200 \mathrm{mg} / \mathrm{L}$ alginate with $1 \mathrm{mM} \mathrm{CaCl} 2$ for all fouling experiments; crossflow velocities and temperatures of feed and draw solutions were $8.5 \mathrm{~cm} / \mathrm{s}$ and $20.0 \pm 0.1^{\circ} \mathrm{C}$, respectively. In FO, the draw solution was $2.5 \mathrm{M}$ glucose. In RO, the applied pressure was $12.5 \mathrm{bar}$ (180 psi). In PFO, the draw solution was $1.5 \mathrm{M}$ glucose, and the applied pressure was 5.5 bar ( 80 psi). Cleaning conditions for FO, PFO, and RO were: crossflow velocity of $17 \mathrm{~cm} / \mathrm{s}$; DI water cleaning solution; and 30 minutes cleaning duration. 
In order to identify whether the different driving forces could affect membrane fouling and subsequent physical cleaning, we compared water flux decline and recovery of these three membrane processes. The water flux decline profiles for FO, RO, and PFO membrane filtration were practically the same (Figure 1A). However, the flux recoveries of these three processes are markedly different (Figure 1B). After the physical cleaning, water flux was almost completely recovered in FO, while by contrast, $\mathrm{RO}$ recovered less than $10 \%$ of the initial water flux decline. PFO, the process which combines osmotic and hydraulic pressures as the driving force, recovered $58 \%$ of the declined water flux, an amount in between FO and RO water flux recoveries.

These observations imply that although the hydraulic pressure exerted less impact on the membrane fouling rate under the reported experimental conditions, it led to significant difference in fouling layer reversibility as observed by the negligible water flux recovery in RO after physical cleaning compared to the near complete water flux recovery in FO. Previous studies attributed such results to the structure of the fouling layer, indicating that fouling layers formed in FO are less compact due to the lack of hydraulic pressure [7, 8]. This hypothesis is strengthened by our observations of increased water flux recovery in RO, PFO, and FO as the contribution of hydraulic pressure to the overall driving force decreased. In FO, the fouling layer is not compacted due to the lack of hydraulic pressure, and the fouled membrane can be easily cleaned by a brief water rinsing. In contrast, the alginate fouling layer on the membrane surface in RO is more compacted and tightly held together under the hydraulic pressure, thereby resulting in a reduced water flux recovery. In PFO, the alginate fouling layer is expected to be partially compressed, which leads to an intermediate water flux recovery.

\subsection{Membrane fouling layer characteristics}

We have used CLSM to characterize the alginate fouling layers formed in FO, RO, and PFO in the hydrated state, for the first time, to verify the effect of hydraulic pressure on organic fouling layer structure. To compare the reversibility of the fouling layers, membrane sections were taken both at the conclusion of the fouling experiments and after physical cleaning, and imaged by CLSM after staining with fluorophore-conjugated Con A as described in Section 2.4. 

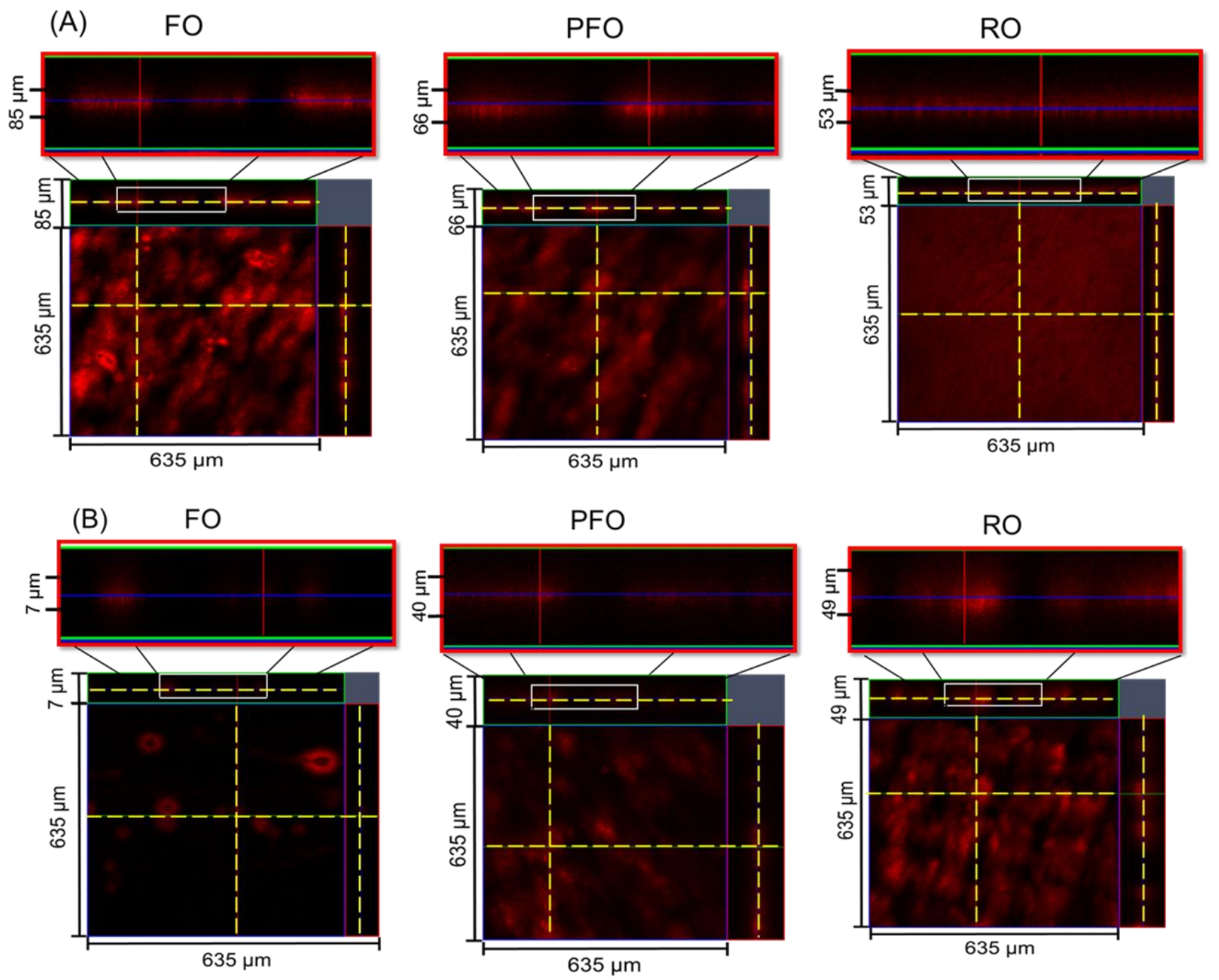

Figure 2: Confocal laser scanning microscopy (CLSM) orthogonal view of (A) alginate fouling layer structures in FO, PFO, and RO; and (B) corresponding alginate fouling layers after physical cleaning. The alginate fouling layer was stained with Con A (red) specific for polysaccharides. Experimental conditions were described in Figure 1. 

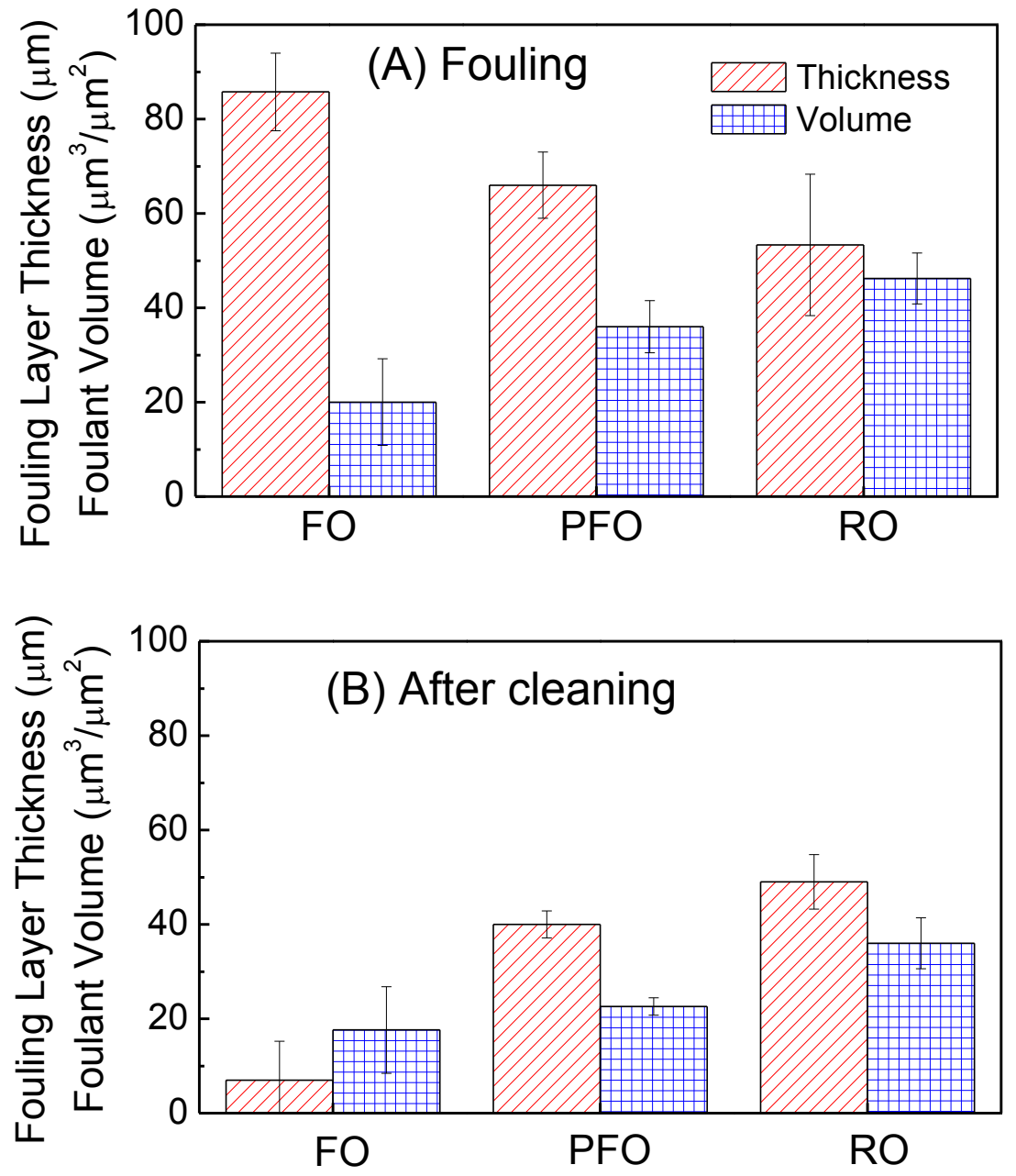

Figure 3: Fouling layer thickness and foulant volume after (A) alginate fouling and (B) physical cleaning in FO, RO, and PFO. The fouling layer thickness and volume were measured with the fouling layers being in the hydrated state (i.e., not dried) by confocal laser scanning microscopy. Error bars represent the standard deviation of twenty measurements in two repeated experiments. Experimental conditions were described in Figure 1. 
Hydraulic pressure resulted in a dramatic difference in alginate fouling layer morphology (Figure 2A). Visually, the fouling layers in RO were homogenous and continuous films, while those in both FO and PFO were highly scattered and heterogeneous. Specifically, average fouling layer thickness gradually decreased from FO $(85 \mu \mathrm{m})$, PFO $(66 \mu \mathrm{m})$, to RO $(53 \mu \mathrm{m})$ (Figure 3A). More importantly, the foulant volume of the alginate fouling layer significantly increased from FO $\left(20 \mu \mathrm{m}^{3} / \mu \mathrm{m}^{2}\right)$, PFO $\left(35 \mu \mathrm{m}^{3} / \mu \mathrm{m}^{2}\right)$, to RO $\left(49 \mu \mathrm{m}^{3} / \mu \mathrm{m}^{2}\right)$ (Figure 3A). The fouling layer thickness and foulant volume captured in the hydrated state were consistent with our hypothesis that the hydraulic pressure compressed the alginate gel, resulting in denser and more compact organic fouling layers.

Our findings regarding the structure of the fouling layers were further corroborated by the fouling layer structures of FO, PFO, and RO after physical cleaning (Figure 2B) by a doubled cross-flow rate for 30 minutes. After the cleaning process, most of the fouling layer in FO was removed, leaving a thin and highly scattered residual layer (largest thickness of $7 \mu \mathrm{m}$ ). However, the fouling layer in RO was only partially disrupted, resulting in heterogeneous film with an average thickness of $49 \mu \mathrm{m}$, almost unchanged from $53 \mu \mathrm{m}$ before cleaning. It is noteworthy that the alginate fouling layer formed in the PFO could not be fully cleaned, leaving a partially covered membrane surface.

Figure 3B clearly shows that the thickness and alginate foulant volume of the remaining alginate fouling layers were dependent on the contribution of hydraulic pressure to the overall driving force. Specifically, both fouling layer thickness and alginate foulant volume after cleaning were largest in RO, lowest in FO, and in between for PFO. This trend also agrees with the estimated alginate fouling layer density after membrane fouling and cleaning as described in Supplementary Data (S3 and Figure S3). Once again, this observation is consistent with our proposal that the alginate fouling layers were compressed under hydraulic pressure, resulting in a varying degree of resistance to physical cleaning.

\subsection{Role of pressure in membrane fouling in FO and RO}

As demonstrated in our experiments, the fouling layers formed on the membranes in RO are irreversible and more compacted than those in FO under identical operational conditions (type of membrane, feedwater composition, hydrodynamic conditions, and initial permeate water flux). We discuss two possible mechanisms for the compaction of the fouling layers in RO: (i) 
permeate drag force across the fouling layer and (ii) compression of foulants under hydraulic pressure.

(A)

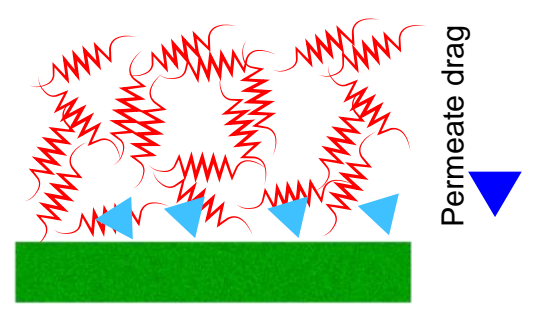

(B)

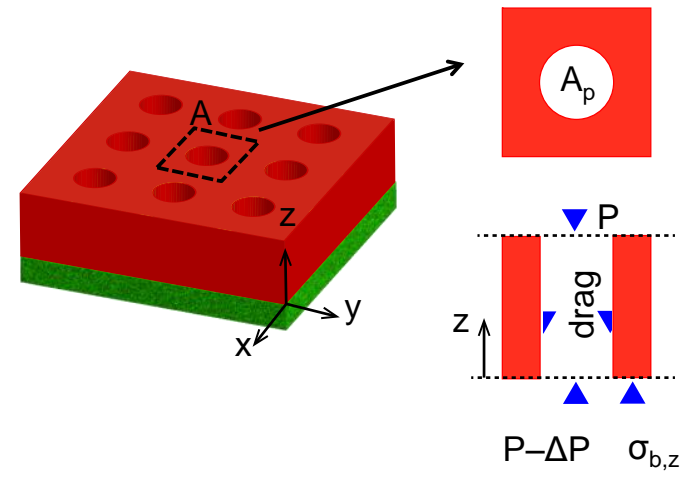

Figure 4: Schematic diagrams of possible compaction mechanisms of fouling layers under hydraulic pressure. (A) Drag force due to viscous flow of water through the fouling layer. (B) Compression of foulants by hydrostatic pressure. Fouling layers are modeled as a polymer matrix consisting of cylindrical pores. Dotted area indicates a repeating unit of the matrix with area $A$ and pore area $A_{p}$ (upper right). Also presented is the cross-section view of the repeating unit showing pressure, stress distribution, and permeate drag force inside the pore (lower right).

\subsubsection{Permeate drag force}

Fouling layers on membranes pose additional resistance to water transport by providing tortuous and porous structures. The porous structure of alginate gel with an effective pore size of $5-150 \mathrm{~nm}[34,35]$ allows the water to transport via viscous flow [36], resulting in a pressure drop across the fouling layer. The corresponding drag force leads to a compressive force on the alginate gel structure in the flow direction (Figure 4A).

The compaction of the fouling layers is solely determined by the pore structures and permeate flow rate of water, not by the absolute magnitude of hydraulic pressure. At the initial stage of fouling, alginate interacts with the membrane surface and starts to deposit, regardless of 
the presence or absence of hydraulic pressure. As additional alginate molecules are transported from the bulk feed water to the membrane surface via the convective permeate water flux, an alginate fouling layer forms through calcium bridging of alginate molecules [26]. Since the initial water fluxes were similar in our RO and FO experiments, the advection of alginate to the membrane surface and the rate of alginate deposition at the initial stage of fouling should be similar in RO and FO. Given the identical initial structures of the fouling layers and water fluxes in both RO and FO, the compaction induced by the permeate drag force at the initial stage of fouling should occur to the same extent. Therefore, the experimentally observed higher degree of compaction in RO than FO cannot be explained by the permeate flow drag force and calls for exploration of additional mechanisms.

\subsubsection{Compression of foulants}

Materials are deformed reversibly or irreversibly under external pressure. One of the most important material properties for such deformation is the Poisson ratio, $v$, defined as the negative ratio of transverse to axial strain. For any compressible foulant in the feed water $(v<$ 0.5), the volume of the foulants is reduced under a large hydraulic pressure in RO. Although most polymers, including alginate gel matrix, are almost incompressible [37, 38], even slight

compressibility of foulants may contribute to compaction to the same degree as that induced by the drag force across fouling layers. To illustrate this effect, we simplified the fouling layers as an impermeable foulant matrix with cylindrical pores distributed periodically (Figure 4B). Each repeating unit of the matrix is assumed to have one pore. Although real fouling layers are highly tortuous and of irregular shape, this simplification can provide insights into the compression of fouling layers.

Assuming the shear stress on the side surfaces of the entire cake is negligible, the compressive normal stress at the bottom of the fouling layer, $\sigma_{\mathrm{b}, \mathrm{z}}$, can be obtained from a force balance on a repeating unit as follows (Figure 4B):

$$
\sigma_{b, z}=P+\Delta P \frac{A_{p}}{A-A_{p}}
$$

where $A_{\mathrm{p}}$ is the pore cross-section area, $A$ the top (and bottom) surface area of the repeating unit which includes $A_{\mathrm{p}}, P$ the hydraulic pressure at the top surface, and $\Delta P$ is the pressure drop across 
the fouling layer. The strain in the flow direction at the bottom of the layer, $\varepsilon_{\mathrm{b}, \mathrm{z}}$, can then be given as

$$
\begin{aligned}
\varepsilon_{b, z} & =\frac{1}{E}\left[\sigma_{b, z}-v\left(\sigma_{b, x}+\sigma_{b, y}\right)\right] \\
& =\frac{1}{E}\left[\left(\frac{A}{A-A_{p}}\right) \Delta P+(1-2 v)(P-\Delta P)\right] \\
& \approx \frac{1}{E}\left[\left(\frac{1}{1-A_{p} / A}\right) \Delta P+(1-2 v) P\right]
\end{aligned}
$$

where $E$ is Young's modulus of the foulant matrix. $\sigma_{\mathrm{b}, \mathrm{x}}$ and $\sigma_{\mathrm{b}, \mathrm{y}}$ are compressive stresses at the bottom of the matrix in $x$ and $y$ directions, respectively, and are set equal to the hydraulic pressure on the membrane surface, $P-\Delta P$.

Since the typical pressure drop across fouling layers in RO is relatively small compared to the trans-membrane pressure, i.e., $\Delta P / P \sim O(1-2 \%$ ) [39], an approximation of $P-\Delta P \approx P$ was used. The first term in the bracket indicates strain induced by the permeate drag force $\left(\varepsilon_{\mathrm{drag}}\right)$, as discussed in the previous subsection, while the second term indicates strain induced by compressibility ( $\left.\varepsilon_{\mathrm{comp}}\right)$. Hence, the ratio of $\varepsilon_{\mathrm{drag}}$ to $\varepsilon_{\mathrm{comp}}$ represents the relative contribution from each factor to the compaction of the fouling layer:

$$
\frac{\varepsilon_{\text {drag }}}{\varepsilon_{\text {comp }}}=\frac{1}{\left(1-A_{p} / A\right)(1-2 v)} \frac{\Delta P}{P}
$$

For sparse fouling layers, we can assume $A_{\mathrm{p}} / A \sim 1$, which yields $\varepsilon_{\text {drag }} / \varepsilon_{\text {comp }} \gg 1$ when $v \sim$ 0.49 is assumed for slight compressibility of foulants. In this case, compressibility does not play an important role. On the other hand, for dense fouling layers with $A_{\mathrm{p}} / A<<1$, Eq. (4) leads to $\varepsilon_{\text {drag }} / \varepsilon_{\text {comp }} \sim O(1)$. This result implies that small compressibility may lead to a compaction effect equivalent to that of the drag force, especially for fouling layers that are already highly compacted.

Polymeric gel matrices are typically assumed to be incompressible [37]. However, actual Poisson ratios for gels have rarely been reported in the literature. Wang et al. [35] conducted compression tests on alginate gel microspheres and measured the temporal change in the strain. Although a quantitative value was not obtained, their study suggested that the Poisson ratio of 
alginate gel matrix is very close to 0.5 . We note that this is the only study that attempted to measure the Poisson ratio of alginate gel matrix, by excluding the effect of solvent migration. Poisson ratios of other hydrogel matrices have been reported, e.g., $\sim 0.46$ for polyacrylamide gel [40], $\sim 0.43$ for polyvinyl alcohol gel [41], and $\sim 0.46$ for poly(N-isopropylacrylamide) gel [42]. Assuming the alginate gel matrix has a similar compressibility, it may be possible that the compaction of the fouling layer in RO was partly due to compression of the alginate gel matrix.

\subsubsection{Dense fouling layers in $R O$}

When the pressure is released after an RO fouling experiment, the compressed materials are relaxed and recover their original volume, unless they are irreversibly compressed. In addition, considering that the fouling layers in FO are loose and can be easily removed, it is likely that the drag force does not play a critical role in compaction, even in RO. However, the mechanisms discussed above work simultaneously and may reinforce each other, which can result in irreversible compaction in $\mathrm{RO}$ as opposed to FO where no compressive force exists, other than drag force across the fouling layers.

As shown in Figures 2 and 3, the reduction in fouling layer thickness after cleaning is prominent in FO, while the reduction becomes progressively smaller for PFO and RO. From force balance analysis, it can be seen that the compressive force induced by the permeate drag force is the weakest at the top of the fouling layer and strongest at the bottom. In FO, most of the fouling layer was washed away during the cleaning process and only a small amount of fouling layer on the membrane surface remained, indicating that the drag force was not large enough for the irreversible compaction. In RO, on the other hand, cleaning was not effective for most of the layer, probably because the entire fouling layer, including the top portion, is highly compacted by the drag force bolstered by the other mechanism. Compaction by any mechanism results in a denser structure and reduces the effective pore sizes while increasing tortuosity. This again leads to an increased drag force that compresses the layers more, especially when foulants are compressible $(v<0.5)$. 


\section{Conclusion}

Our results demonstrate that hydraulic pressure plays an important role in membrane organic fouling behavior, particularly in fouling reversibility in FO, PFO, and RO. Visual examination of fouling layers in the hydrated state using CLSM revealed that the fouling layer thickness decreased in the order of FO, PFO, and RO, while the alginate foulant volume increased from FO, PFO, to RO. However, after a brief physical cleaning by increasing the shear flow rate, both the thickness and foulant volume of the remaining alginate fouling layers increased in the order of FO, PFO, and RO. Such fouling layer characteristics indicate that the large hydraulic pressure in RO induces significant compression and compaction of the alginate fouling layer, which leads to irreversible fouling layer formation.

We proposed two possible compaction mechanisms in RO, namely permeate drag force and compression of foulants. Fouling layers in all membrane processes investigated (FO, PFO, and RO) undergo similar extent of compaction due to the permeate drag force as identical initial water fluxes were employed. However, the compressibility of foulants under hydraulic pressure may contribute to compression of fouling layers to a significant extent. Finally, we emphasize that these mechanisms work simultaneously and therefore reinforce each other, resulting in irreversible, dense and compact fouling layers in RO, as opposed to those in FO, where only drag force across the fouling layers is applied as a compressive force.

\section{Acknowledgements}

Financial support from the Department of Defense through the Strategic Environmental Research and Development Program (SERDP, Project ER-2217) is gratefully acknowledged. 


\section{References}

[1] M.A. Shannon, P.W. Bohn, M. Elimelech, J.G. Georgiadis, B.J. Marinas, A.M. Mayes, Science and technology for water purification in the coming decades, Nature, 452 (2008) 301310.

[2] R. Semiat, Energy issues in desalination processes, Environmental Science and Technology, 42 (2008) 8193-8201.

[3] J.R. Stokes, A. Horvath, Energy and Air Emission Effects of Water Supply, Environmental Science \& Technology, 43 (2009) 2680-2687.

[4] S. Zou, Y. Gu, D. Xiao, C.Y. Tang, The role of physical and chemical parameters on forward osmosis membrane fouling during algae separation, Journal of Membrane Science, 366 (2011) 356-362.

[5] S. Lee, M. Elimelech, Relating Organic Fouling of Reverse Osmosis Membranes to Intermolecular Adhesion Forces, Environmental Science \& Technology, 40 (2006) 980-987.

[6] W.S. Ang, S. Lee, M. Elimelech, Chemical and physical aspects of cleaning of organicfouled reverse osmosis membranes, Journal of Membrane Science, 272 (2006) 198-210.

[7] B. Mi, M. Elimelech, Chemical and physical aspects of organic fouling of forward osmosis membranes, Journal of Membrane Science, 320 (2008) 292-302.

[8] B. Mi, M. Elimelech, Organic fouling of forward osmosis membranes: Fouling reversibility and cleaning without chemical reagents, Journal of Membrane Science, 348 (2010) 337-345.

[9] C. Boo, M. Elimelech, S. Hong, Fouling control in a forward osmosis process integrating seawater desalination and wastewater reclamation, Journal of Membrane Science, 444 (2013) 148-156.

[10] S. Lee, C. Boo, M. Elimelech, S. Hong, Comparison of fouling behavior in forward osmosis (FO) and reverse osmosis (RO), Journal of Membrane Science, 365 (2010) 34-39.

[11] J.R.B. Herron, Edward G. Salter, Robert, Direct osmotic concentration contaminated water, in, OSMOTEK, INC., 1997.

[12] R.W. Holloway, A.E. Childress, K.E. Dennett, T.Y. Cath, Forward osmosis for concentration of anaerobic digester centrate, Water Research, 41 (2007) 4005-4014.

[13] A. Achilli, T.Y. Cath, E.A. Marchand, A.E. Childress, The forward osmosis membrane bioreactor: A low fouling alternative to MBR processes, Desalination, 239 (2009) 10-21.

[14] E.R. Cornelissen, D. Harmsen, K.F. de Korte, C.J. Ruiken, J.-J. Qin, H. Oo, L.P. Wessels, Membrane fouling and process performance of forward osmosis membranes on activated sludge, Journal of Membrane Science, 319 (2008) 158-168.

[15] T.Y. Cath, S. Gormly, E.G. Beaudry, M.T. Flynn, V.D. Adams, A.E. Childress, Membrane contactor processes for wastewater reclamation in space: Part I. Direct osmotic concentration as pretreatment for reverse osmosis, Journal of Membrane Science, 257 (2005) 85-98.

[16] M. Xie, L.D. Nghiem, W.E. Price, M. Elimelech, Toward Resource Recovery from Wastewater: Extraction of Phosphorus from Digested Sludge Using a Hybrid Forward Osmosis- 
Membrane Distillation Process, Environmental Science \& Technology Letters, 1 (2014) 191195.

[17] M. Xie, L.D. Nghiem, W.E. Price, M. Elimelech, A Forward Osmosis-Membrane Distillation Hybrid Process for Direct Sewer Mining: System Performance and Limitations, Environmental Science \& Technology, 47 (2013) 13486-13493.

[18] S. Zou, Y.-N. Wang, F. Wicaksana, T. Aung, P.C.Y. Wong, A.G. Fane, C.Y. Tang, Direct microscopic observation of forward osmosis membrane fouling by microalgae: Critical flux and the role of operational conditions, Journal of Membrane Science, 436 (2013) 174-185.

[19] Y. Wang, F. Wicaksana, C.Y. Tang, A.G. Fane, Direct Microscopic Observation of Forward Osmosis Membrane Fouling, Environmental Science \& Technology, 44 (2010) 7102-7109.

[20] Y. Liu, B. Mi, Combined fouling of forward osmosis membranes: Synergistic foulant interaction and direct observation of fouling layer formation, Journal of Membrane Science, 407-408 (2012) 136-144.

[21] P. Le-Clech, Y. Marselina, Y. Ye, R.M. Stuetz, V. Chen, Visualisation of polysaccharide fouling on microporous membrane using different characterisation techniques, Journal of Membrane Science, 290 (2007) 36-45.

[22] M. Ferrando, A. Rŏżek, M. Zator, F. López, C. Güell, An approach to membrane fouling characterization by confocal scanning laser microscopy, Journal of Membrane Science, 250 (2005) 283-293.

[23] M. Zator, M. Ferrando, F. López, C. Güell, Membrane fouling characterization by confocal microscopy during filtration of BSA/dextran mixtures, Journal of Membrane Science, 301 (2007) 57-66.

[24] M. Marroquin, A. Vu, T. Bruce, R. Powell, S.R. Wickramasinghe, S.M. Husson, Location and quantification of biological foulants in a wet membrane structure by cross-sectional confocal laser scanning microscopy, Journal of Membrane Science, 453 (2014) 282-291.

[25] A. Tiraferri, N.Y. Yip, A.P. Straub, S. Romero-Vargas Castrillon, M. Elimelech, A method for the simultaneous determination of transport and structural parameters of forward osmosis membranes, Journal of Membrane Science, 444 (2013) 523-538.

[26] Q. Li, M. Elimelech, Organic Fouling and Chemical Cleaning of Nanofiltration Membranes: Measurements and Mechanisms, Environmental Science \& Technology, 38 (2004) 4683-4693.

[27] C. Boo, S. Lee, M. Elimelech, Z. Meng, S. Hong, Colloidal fouling in forward osmosis: Role of reverse salt diffusion, Journal of Membrane Science, 390-391 (2012) 277-284.

[28] M. Xie, L.D. Nghiem, W.E. Price, M. Elimelech, Impact of humic acid fouling on membrane performance and transport of pharmaceutically active compounds in forward osmosis, Water Research, 47 (2013) 4567-4575.

[29] Y. Mo, A. Tiraferri, N.Y. Yip, A. Adout, X. Huang, M. Elimelech, Improved Antifouling Properties of Polyamide Nanofiltration Membranes by Reducing the Density of Surface Carboxyl Groups, Environmental Science \& Technology, 46 (2012) 13253-13261. 
[30] F.S. Coulibaly, B.-B.C. Youan, Concanavalin A-Polysaccharides binding affinity analysis using a quartz crystal microbalance, Biosensors and Bioelectronics, 59 (2014) 404-411.

[31] E. Bar-Zeev, K. Zodrow, S. Kwan, E. Menachem, The importance of microscopic charaterization of membrane biofilms in an unconfined environment, Desalination, 348 (2014) 815.

[32] L.N. Mueller, J.F. de Brouwer, J.S. Almeida, L.J. Stal, J.B. Xavier, Analysis of a marine phototrophic biofilm by confocal laser scanning microscopy using the new image quantification software PHLIP, BMC ecology, 6 (2006) 1.

[33] M. Kuehn, M. Hausner, H.-J. Bungartz, M. Wagner, P.A. Wilderer, S. Wuertz, Automated Confocal Laser Scanning Microscopy and Semiautomated Image Processing for Analysis of Biofilms, Applied and Environmental Microbiology, 64 (1998) 4115-4127.

[34] K.Y. Lee, D.J. Mooney, Alginate: properties and biomedical applications, Progress in polymer science, 37 (2012) 106-126.

[35] C. Wang, C. Cowen, Z. Zhang, C.R. Thomas, High-speed compression of single alginate microspheres, Chem Eng Sci, 60 (2005) 6649-6657.

[36] Y.Y. Suzuki, M. Tokita, S. Mukai, Kinetics of water flow through a polymer gel, The European physical journal. E, Soft matter, 29 (2009) 415-422.

[37] S.Q. Cai, Y.H. Hu, X.H. Zhao, Z.G. Suo, Poroelasticity of a covalently crosslinked alginate hydrogel under compression, J Appl Phys, 108 (2010).

[38] J.W. Yoon, S.Q. Cai, Z.G. Suo, R.C. Hayward, Poroelastic swelling kinetics of thin hydrogel layers: comparison of theory and experiment, Soft Matter, 6 (2010) 6004-6012.

[39] E.M. Hoek, M. Elimelech, Cake-enhanced concentration polarization: a new fouling mechanism for salt-rejecting membranes, Environmental science \& technology, 37 (2003) 55815588.

[40] T. Takigawa, Y. Morino, K. Urayama, T. Masuda, Poisson's ratio of polyacrylamide (PAAm) gels, Polymer Gels and Networks, 4 (1996) 1-5.

[41] K. Urayama, T. Takigawa, T. Masuda, Poisson's ratio of poly(vinyl alcohol) gels, Macromolecules, 26 (1993) 3092-3096.

[42] T. Takigawa, T. Ikeda, Y. Takakura, T. Masuda, Swelling and stress-relaxation of poly(Nisopropylacrylamide) gels in the collapsed state, J Chem Phys, 117 (2002) 7306-7312.

[43] J.G. Wijmans, R.W. Baker, The solution-diffusion model: a review, Journal of Membrane Science, 107 (1995) 1-21.

[44] M. Soltanieh, T. Zaare-Asl, A modified solution-diffuison model for separation of ethanolwater azeotropic mixtures in pervaporation, Chemical Engineering Communications, 152-153 (1996) 405-412.

[45] M. Soltanieh, W.N. Gill, Review of Reverse-Osmosis Membranes and Transport Models, Chem Eng Commun, 12 (1981) 279-363.

[46] G. Belfort, A molecular friction model for transport of uncharged solutes in neutral hyperfiltration and ultrafiltration membranes containing bound water, Desalination, 18 (1976) 259-281. 
[47] M.N. Sarbolouki, Reverse osmosis and synthetic membranes - theory, technology, engineering, S. Sourirajan, Ed., National Research Council of Canada, Ottawa, Canada, 1977, 598 pp. \$45.00, Journal of Polymer Science: Polymer Letters Edition, 15 (1977) 629-630.

[48] T.K. Sherwood, P.L.T. Brian, R.E. Fisher, Desalination by Reverse Osmosis, Industrial \& Engineering Chemistry Fundamentals, 6 (1967) 2-12.

[49] L. Applegate, C.R. Antonson, The Phenomenological Characterization of DP-1 Membranes, in: H.K. Lonsdale, H.E. Podall (Eds.) Reverse Osmosis Membrane Research, Springer US, 1972, pp. 243-252.

[50] J.M. Dickson, T. Matsuura, P. Blais, S. Sourirajan, Some Transport Characteristics of Aromatic Polyamide Membranes in Reverse-Osmosis, J Appl Polym Sci, 20 (1976) 1491-1499.

[51] U. Merten, Desalination by reverse osmosis, M.I.T. Press, Cambridge,, 1966.

[52] T.D. Nguyen, K. Chan, T. Matsuura, S. Sourirajan, Effect of Shrinkage on Pore-Size and Pore-Size Distribution of Different Cellulosic Reverse-Osmosis Membranes, Ind Eng Chem Prod Rd, 23 (1984) 501-508.

[53] K. Kosutic, B. Kunst, RO and NF membrane fouling and cleaning and pore size distribution variations, Desalination, 150 (2002) 113-120.

[54] S.H. Kim, S.Y. Kwak, T. Suzuki, Positron annihilation spectroscopic evidence to demonstrate the flux-enhancement mechanism in morphology-controlled thin-film-composite (TFC) membrane, Environ Sci Technol, 39 (2005) 1764-1770.

[55] T.D. Nguyen, T. Matsuura, S. Sourirajan, Effect of Nonsolvent Additives on the Pore-Size and the Pore-Size Distribution of Aromatic Polyamide Ro Membranes, Chem Eng Commun, 54 (1987) 17-36.

[56] F. Wang, V.V. Tarabara, Pore blocking mechanisms during early stages of membrane fouling by colloids, Journal of Colloid and Interface Science, 328 (2008) 464-469.

[57] J.T. Duan, E. Litwiller, I. Pinnau, Solution-diffusion with defects model for pressureassisted forward osmosis, J Membrane Sci, 470 (2014) 323-333. 


\section{Graphical Abstract}
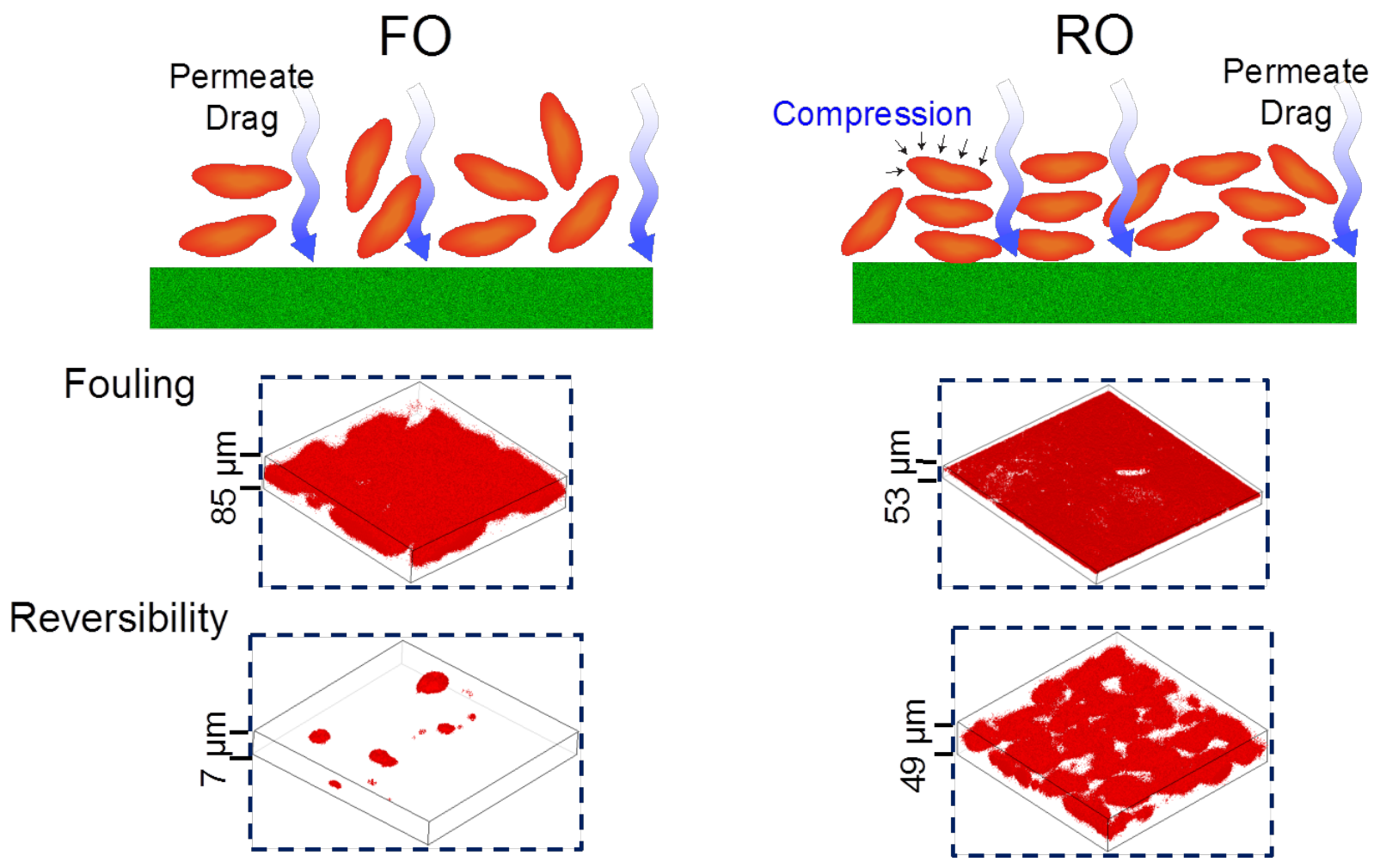